\title{
Protease inclusion in plant- and animal-based broiler diets: Performance, digestibility and biometry of digestive organs
}

\author{
D.P. Carvalho', N.S.M. Leandro', M.A. Andrade', H.F. de Oliveira ${ }^{1 \#}$, M.F. Pires ${ }^{1}$, K.A. \\ Teixeira', P.S. Assunção ${ }^{1}$ \& J.H. Stringhini ${ }^{1}$ \\ ${ }^{1}$ Federal University of Goiás, Department of Animal Science, Goiânia, Goiás, Brazil
}

(Received 17 October 2019; Accepted 25 March 2020; First published online 18 May 2020)

\author{
Copyright resides with the authors in terms of the Creative Commons Attribution 4.0 South African License. \\ See: http://creativecommons.org/licenses/by/4.0/za \\ Condition of use: The user may copy, distribute, transmit and adapt the work, but must recognize the authors and the South African \\ Journal of Animal Science.
}

\begin{abstract}
The addition of protease to broiler diets may complement the action of endogenous enzymes and improve protein digestibility. Here, the authors evaluated the effect of adding protease to broiler diets that contained animal-based meal on bird performance, digestibility, and biometry of digestive tract organs. Four treatments, which contained animal- or plant-based meals with or without supplementary protease were compared, namely basal vegetable feed (BVF), BVF + protease (BFP), basal vegetable feed + animal byproduct meal (BFA), and BFA + protease. In the first experiment, 320 one-day-old Cobb $500 \AA$ chicks were allocated to eight replicates with 10 birds per replicate. The experimental period was seven days, and nutrient metabolizability was evaluated. In the second experiment, 720 one-day-old Cobb $500 \AA$ chicks were assigned to treatments in a similar manner, with six replicates and 30 birds per replicate, but the experimental period was 42 days. Significant differences $(P<0.05)$ were observed between treatments for nutrient digestibility, weight gain, feed consumption, average final weight, food conversion, viability, and biometry of the pancreas. Treatments with animal-based meals had the highest digestibility. Birds fed these meals grew faster in the pre-starter phase and consumed less feed between 1 and 21 days. However, between 1 and 42 days old, broilers fed plant-based diets had better feed conversion, and the group that did not receive protease supplementation had a better liveability rate.
\end{abstract}

Keywords: additive, exogenous enzyme, nutrition, organ biometry, poultry

\#Corresponding author: helder@zootecnista.com.br

\section{Introduction}

Broiler production in Brazil is growing fast, and the country has become the world's second largest producer and the greatest exporter. In 2017, production of the poultry sector was approximately 13.05 million tons (BAAP, 2018).

Broiler production provides quality animal protein at low cost and is accessible to most social classes (Oliveira et al., 2018). Because nearly $70 \%$ of all costs in broiler production are related to feeding, there is a need to promote the use of alternative ingredients in their diets (Wachholz et al., 2017). The increase in chicken production and resultant increase in residues produced by the sector have led to higher production of animal by-product meals, which can be considered an alternative source of nutrients to reduce broiler production costs by partly replacing soybean bran. The inclusion of such by-products as animal meals has the potential to improve efficiency in the use of traditional foods.

However, besides corn and soybean bran, the majority of foods used in diets contain anti-nutritional factors such as protease inhibitors and phytates, which reduce food quality and inhibit nutrient digestibility for chickens. In this context, Matias et al. (2015) suggested the use of exogenous enzymes as an alternative way to reduce production costs, by improving efficiency in the use of traditional foods and enabling the use of animal by-product meals. Among the exogenous enzymes, proteases have been prominent, as they promote higher protein digestibility of the ingredients in feeds by hydrolysing them into peptides and amino acids, thus favouring their absorption (Ribeiro et al., 2015).

Protease supplementation is of particular interest for very young animals, for which the relative activity of endogenous proteases may not be optimal. Endogenous proteases catalyse the hydrolysis of dietary 
proteins, thereby complementing the animals' digestive enzymes, such as pepsin and pancreatic proteases, but they also destroy anti-nutrients, such as lectins and trypsin inhibitors. Exogenous proteases are thought not to have this effect (Ghazi et al., 2002; Cowieson et al., 2016).

The present study evaluated the effects of adding protease enzyme $(0.05 \%)$ to broiler diets containing animal meal. The authors measured nutrient digestibility, performance, and the biometry of digestive tract organs for chicks aged between 1 and 42 days.

\section{Material and Methods}

The authors conducted two experiments at the Poultry Farming Sector of the Zootechny Department of the Federal University of Goiás. All procedures in this study were conducted according to protocol registration No. 055/15, and were approved by the Ethics Committee for Animal Use of the Federal University of Goiás.

For the first experiment, 320 one-day-old male Cobb $500 \AA$ chicks with an average initial weight of $48 \pm$ $2 \mathrm{~g}$ were distributed in a $2 \times 2$ factorial arrangement by a completely randomized design, providing four treatments with eight replicates and 10 birds per replicate. Treatments comprised feeds containing animal- or plant-based meals with or without supplementary protease enzyme: i) basal vegetable feed (BVF), ii) BVF + protease (BFP), iii) basal vegetable feed + animal by-product meal (BFA), and iv) BFA + protease. The experimental period consisted of three days for adaptation and four days for data collection. Experimental diets were formulated according to the nutritional requirements proposed by Rostand et al. (2011), modified by a reduction of $7 \%$ in all amino acids, $50 \mathrm{kcal}$ of energy, and use of the protease enzyme as a substitute for starch. Diets contained no growth promoters or anticoccidians. Nutritional and percentage composition are shown in Tables 1 to 3 .

Birds were housed in batteries of 32 experimental cages, each $0.40 \times 0.50 \mathrm{~m}$ in size and constructed with galvanized wire. Each was equipped with manual drinkers and feeders, and an excreta-collecting tray. Ventilation was controlled with curtains. Feed and water were provided ad libitum for the entire experimental period. The sheds were monitored for temperature and relative air humidity. Lighting was constant with the use of incandescent lamps.

Metabolism was assayed from the fourth to the seventh day, using total excreta collection (Sakomura \& Rostagno, 2007). Excreta were collected twice a day, and samples were stored in identified plastic bags and frozen. For bromatological analyses, samples were pre-dried in a forced ventilation oven at $55 \pm 5{ }^{\circ} \mathrm{C}$ and then ground in a Wiley mill. Analyses were performed according to the methods proposed by Silva and Queiroz (2002). The authors calculated nutritional balances as proposed by Matterson et al. (1965), and metabolizability coefficients as proposed by Batal and Parsons (2002). The nutrient metabolizability (M) was determined by the equation:

Where: $\mathrm{NI}=$ ingested nutrient, and

$$
M(\%)=\frac{N I-N E}{N I} \times 100
$$

$\mathrm{NE}=$ excreted nutrient.

For the second experiment, 720 one-day-old male Cobb $500 \circledast$ chicks, with an average initial weight of $43 \pm 1 \mathrm{~g}$, were distributed in a $2 \times 2$ factorial arrangement by a completely randomized design, providing four treatments with six replicates and 30 birds per replicate. Treatments were the same as those used in the first experiment, but the experimental period was 42 days, divided into four phases, namely pre-initial, initial, growth, and termination. Experimental diets were formulated as before, and diets contained no antibiotics or anticoccidians. Percentage and nutritional compositions are shown in Table 2 and Table 3.

Birds were reared on the floor, stored in 24 experimental concrete boxes $(2.10 \times 2.50 \mathrm{~m})$, each equipped with pendular drinkers and feeders, and rice hull litter. Birds were reared under the same management conditions as the first experiment. At 7, 14, 21, 34, and 42 days, the authors evaluated feed intake $(\mathrm{g})$, average weight $(\mathrm{g})$, weight gain $(\mathrm{g})$, food conversion $(\mathrm{kg} / \mathrm{kg})$, and liveability $(\%)$. Feed intake $(\mathrm{g})$ was calculated as the weight difference between the feed provided and the leftovers. Average weight was calculated by the total weight of chickens in the batch, divided by the number of chickens. Weight gain was obtained through the difference between birds' initial average weight and final average weight. Feed conversion was calculated as the relationship between weight gain and feed consumption. To calculate feed consumption and feed conversion variables, the authors considered mortality rates, which were recorded daily. Liveability was determined through the equation:

$$
\text { Livability }(\%)=\frac{\text { final number birds } x 100}{\text { initial number of birds }}
$$


Table 1 Composition of experimental feeds containing protease and animal by-product meals for broilers in the pre-starter phase, days 1 to 7

\begin{tabular}{|c|c|c|c|c|}
\hline \multirow{2}{*}{ Ingredients, g/kg } & \multicolumn{4}{|c|}{ Treatments } \\
\hline & $\mathrm{BF}$ & BFP & BFA & BFAP \\
\hline Corn & 60.13 & 60.13 & 65.31 & 65.31 \\
\hline Soybean meal & 35.53 & 35.53 & 24.41 & 24.41 \\
\hline Viscera meal & & & 3.73 & 3.73 \\
\hline Feather meal & & & 1.67 & 1.67 \\
\hline Meat and bone meal & & & 2.00 & 2.00 \\
\hline Dicalcium phosphate & 1.89 & 1.89 & 0.76 & 0.76 \\
\hline Starch & 0.10 & 0.05 & 0.10 & 0.05 \\
\hline Protease & & 0.05 & & 0.05 \\
\hline Limestone & 1.04 & 1.04 & 0.64 & 0.64 \\
\hline Vitamin supplement ${ }^{1}$ & 0.10 & 0.10 & 0.10 & 0.10 \\
\hline Mineral supplement ${ }^{2}$ & 0.05 & 0.05 & 0.05 & 0.05 \\
\hline Salt & 0.45 & 0.45 & 0.36 & 0.36 \\
\hline DL-Methionine & 0.37 & 0.37 & 0.35 & 0.35 \\
\hline L-Lysine & 0.24 & 0.24 & 0.38 & 0.38 \\
\hline L-Threonine & 0.08 & 0.08 & 0.09 & 0.09 \\
\hline \multicolumn{5}{|l|}{ Calculated composition, \% } \\
\hline Metabolizable energy, Kcal/kg & 2900 & 2900 & 2900 & 2900 \\
\hline Crude protein & 21.85 & 21.85 & 21.85 & 21.85 \\
\hline Digestible threonine & 0.79 & 0.79 & 0.79 & 0.79 \\
\hline Digestible methionine+cysteine & 0.95 & 0.95 & 0.94 & 0.94 \\
\hline Digestible methionine & 0.66 & 0.66 & 0.64 & 0.64 \\
\hline Digestible lysine & 1.21 & 1.21 & 1.21 & 1.21 \\
\hline Calcium & 1.00 & 1.00 & 0.93 & 0.93 \\
\hline Available phosphorus & 0.46 & 0.46 & 0.47 & 0.47 \\
\hline Sodium & 0.22 & 0.22 & 0.22 & 0.22 \\
\hline
\end{tabular}

BVF: basal vegetable feed, BFP: BVF + protease, BFA: basal vegetable feed + animal by-product meal, BFAP: BFA + protease (BFAP). ${ }^{1}$ selenium $0.30 \mathrm{mg}$, vitamin $\mathrm{A} 10.000 \mathrm{Ul}$, vitamin $\mathrm{D}_{3} 2.500 \mathrm{Ul}$, vitamin E $25 \mathrm{mg}$, vitamin $\mathrm{K}_{3} 2 \mathrm{mg}$, vitamin $B_{1} 2.50 \mathrm{mg}$, vitamin $B_{2} 6.50 \mathrm{mg}$, vitamin $B_{6} 3.50 \mathrm{mg}$, vitamin $B_{12} 18 \mathrm{mcg}$, folic acid $1.20 \mathrm{mg}$, pantothenic acid 15 $\mathrm{mg}$, niacin $42 \mathrm{mg}$, biotin $80 \mathrm{mcg}$, ethoxyquin $166 \mathrm{mg}$

${ }^{2}$ manganese $90 \mathrm{mg}$, zinc $75 \mathrm{mg}$, iron $60 \mathrm{mg}$, copper $9.75 \mathrm{mg}$, iodine $1.20 \mathrm{mg}$ 
Table 2 Composition of experimental feeds containing protease and animal by-product meals in pre-starter ( 1 to 7 days) and starter ( 8 to 21 days) phases for broilers

\begin{tabular}{|c|c|c|c|c|c|c|c|c|}
\hline \multirow{3}{*}{ Ingredients, g/kg } & \multicolumn{8}{|c|}{ Treatments } \\
\hline & \multicolumn{4}{|c|}{ Pre-starter phase } & \multicolumn{4}{|c|}{ Starter phase } \\
\hline & $\mathrm{BF}$ & BFP & BFA & BFAP & $\mathrm{BF}$ & BFP & BFA & BFAP \\
\hline Corn & 60.13 & 60.13 & 65.31 & 65.31 & 63.26 & 63.26 & 67.62 & 67.62 \\
\hline Soybean meal & 35.53 & 35.53 & 24.41 & 24.41 & 32.92 & 32.92 & 23.71 & 23.71 \\
\hline Viscera meal & & & 3.73 & 3.73 & & & 2.58 & 2.58 \\
\hline Feather meal & & & 1.67 & 1.67 & & & 1.00 & 1.00 \\
\hline Meat and bone meal & & & 2.00 & 2.00 & & & 3.03 & 3.03 \\
\hline Dicalcium phosphate & 1.89 & 1.89 & 0.76 & 0.76 & 1.76 & 1.76 & 0.47 & 0.47 \\
\hline Soy oil & & & & & 0.20 & 0.20 & & \\
\hline Starch & 0.10 & 0.05 & 0.10 & 0.05 & 0.10 & 0.05 & 0.10 & 0.05 \\
\hline Protease & & 0.05 & & 0.05 & & 0.05 & & 0.05 \\
\hline Limestone & 1.04 & 1.04 & 0.64 & 0.64 & 0.82 & 0.82 & 0.54 & 0.54 \\
\hline Vitamin supplement ${ }^{1}$ & 0.10 & 0.10 & 0.10 & 0.10 & 0.10 & 0.10 & 0.10 & 0.10 \\
\hline Mineral supplement ${ }^{2}$ & 0.05 & 0.05 & 0.05 & 0.05 & 0.05 & 0.05 & 0.05 & 0.05 \\
\hline Salt & 0.45 & 0.45 & 0.36 & 0.36 & 0.43 & 0.43 & 0.35 & 0.35 \\
\hline DL-Methionine & 0.37 & 0.37 & 0.35 & 0.35 & 0.24 & 0.24 & 0.24 & 0.24 \\
\hline L-Lysine & 0.24 & 0.24 & 0.38 & 0.38 & 0.09 & 0.09 & 0.20 & 0.20 \\
\hline L-Threonine & 0.08 & 0.08 & 0.09 & 0.09 & & & & \\
\hline \multicolumn{9}{|l|}{ Calculated composition, $\%$} \\
\hline Metabolizable energy, Kcal/kg & 2900 & 2900 & 2900 & 2900 & 2950 & 2950 & 2950 & 2950 \\
\hline Crude protein & 21.85 & 21.85 & 21.85 & 21.85 & 20.65 & 20.65 & 20.65 & 20.65 \\
\hline Digestible threonine & 0.79 & 0.79 & 0.79 & 0.79 & 0.69 & 0.69 & 0.67 & 0.67 \\
\hline Digestible methionine+cysteine & 0.95 & 0.95 & 0.94 & 0.94 & 0.80 & 0.80 & 0.80 & 0.80 \\
\hline Digestible methionine & 0.66 & 0.66 & 0.64 & 0.64 & 0.53 & 0.53 & 0.53 & 0.53 \\
\hline Digestible lysine & 1.21 & 1.21 & 1.21 & 1.21 & 1.03 & 1.03 & 1.03 & 1.03 \\
\hline Calcium & 1.00 & 1.00 & 0.93 & 0.93 & 0.87 & 0.87 & 0.87 & 0.87 \\
\hline Available phosphorus & 0.46 & 0.46 & 0.47 & 0.47 & 0.44 & 0.44 & 0.44 & 0.44 \\
\hline Sodium & 0.22 & 0.22 & 0.22 & 0.22 & 0.21 & 0.21 & 0.21 & 0.21 \\
\hline
\end{tabular}

BVF: basal vegetable feed, BFP: BVF + protease, BFA: basal vegetable feed + animal by-product meal, BFAP: BFA + protease

${ }^{1}$ Selenium $0.30 \mathrm{mg}$, vitamin A $10.000 \mathrm{UI}$, vitamin D3 $2.500 \mathrm{UI}$, vitamin E $25 \mathrm{mg}$. vitamin K3 $2 \mathrm{mg}$, vitamin B1 $2.50 \mathrm{mg}$, vitamin B2 $6.50 \mathrm{mg}$, vitamin B6 $3.50 \mathrm{mg}$, vitamin B12 $18 \mathrm{mcg}$, folic acid $1.20 \mathrm{mg}$, pantothenic acid $15 \mathrm{mg}$, niacin $42 \mathrm{mg}$, biotin $80 \mathrm{mcg}$, ethoxyquin $166 \mathrm{mg}$

${ }^{2}$ Manganese $90 \mathrm{mg}$, zinc $75 \mathrm{mg}$, iron $60 \mathrm{mg}$, copper $9.75 \mathrm{mg}$, iodine $1.20 \mathrm{mg}$ 
Table 3 Composition of experimental feeds containing protease and animal by-product meals in growing (22 to 35 days) and final ( 36 to 42 days) for broilers

\begin{tabular}{|c|c|c|c|c|c|c|c|c|}
\hline \multirow{3}{*}{ Ingredients, g/kg } & \multicolumn{8}{|c|}{ Treatments } \\
\hline & \multicolumn{4}{|c|}{22 to 35 days } & \multicolumn{4}{|c|}{36 to 42 days } \\
\hline & $\mathrm{BF}$ & BFP & BFA & BFAP & $\mathrm{BF}$ & BFP & BFA & BFAP \\
\hline Corn & 66.91 & 66.91 & 72.49 & 72.49 & 69.40 & 69.40 & 73.64 & 73.64 \\
\hline Soybean meal & 28.59 & 28.59 & 17.95 & 17.95 & 26.04 & 26.04 & 17.97 & 17.97 \\
\hline Viscera meal & - & - & 2.00 & 2.00 & - & - & 2.59 & 2.59 \\
\hline Feather meal & - & - & 2.67 & 2.67 & - & - & 1.00 & 1.00 \\
\hline Meat and bone meal & - & - & 2.00 & 2.00 & - & - & 1.00 & 1.00 \\
\hline Dicalcium phosphate & 1.60 & 1.60 & 0.67 & 0.67 & 1.45 & 1.45 & 0.79 & 0.79 \\
\hline Soy oil & 0.99 & 0.99 & 0.54 & 0.54 & 1.25 & 1.25 & 1.20 & 1.20 \\
\hline Starch & 0.10 & 0.05 & 0.10 & 0.05 & 0.10 & 0.05 & 0.10 & 0.05 \\
\hline Protease & - & 0.05 & - & 0.05 & - & 0.05 & - & 0.05 \\
\hline Limestone & 0.78 & 0.78 & 0.63 & 0.63 & 0.75 & 0.75 & 0.63 & 0.63 \\
\hline Vitamin supplement ${ }^{1}$ & 0.10 & 0.10 & 0.10 & 0.10 & 0.10 & 0.10 & 0.10 & 0.10 \\
\hline Mineral supplement ${ }^{2}$ & 0.05 & 0.05 & 0.05 & 0.05 & 0.05 & 0.05 & 0.05 & 0.05 \\
\hline Salt & 0.41 & 0.41 & 0.33 & 0.33 & 0.38 & 0.38 & 0.33 & 0.33 \\
\hline DL-Methionine & 0.24 & 0.24 & 0.13 & 0.13 & 0.26 & 0.26 & 0.26 & 0.26 \\
\hline L-Lysine & 0.20 & 0.20 & 0.32 & 0.32 & 0.22 & 0.22 & 0.28 & 0.28 \\
\hline L-Threonine & - & - & 0.01 & 0.01 & - & - & 0.02 & 0.02 \\
\hline \multicolumn{9}{|l|}{ Calculated composition, \% } \\
\hline Metabolizable energy Kcal/kg & 3050 & 3050 & 3050 & 3050 & 3100 & 3100 & 3100 & 3100 \\
\hline Crude protein & 19.10 & 19.10 & 19.10 & 19.10 & 18.16 & 18.16 & 17.74 & 17.74 \\
\hline Digestible threonine & 0.63 & 0.63 & 0.63 & 0.63 & 0.60 & 0.60 & 0.60 & 0.60 \\
\hline Digestible methionine+cysteine & 0.77 & 0.77 & 0.67 & 0.67 & 0.77 & 0.77 & 0.77 & 0.77 \\
\hline Digestible methionine & 0.51 & 0.51 & 0.39 & 0.39 & 0.52 & 0.52 & 0.51 & 0.51 \\
\hline Digestible lysine & 1.02 & 1.02 & 0.97 & 0.97 & 0.97 & 0.97 & 0.92 & 0.92 \\
\hline Calcium & 0.81 & 0.81 & 0.81 & 0.81 & 0.75 & 0.75 & 0.75 & 0.75 \\
\hline Available phosphorus & 0.40 & 0.40 & 0.40 & 0.40 & 0.37 & 0.37 & 0.37 & 0.37 \\
\hline Sodium & 0.20 & 0.20 & 0.20 & 0.20 & 0.19 & 0.19 & 0.19 & 0.19 \\
\hline
\end{tabular}

BVF: basal vegetable feed, BFP: BVF + protease, BFA: basal vegetable feed + animal by-product meal, BFAP: BFA + protease

${ }_{1}^{1}$ Selenium $0.30 \mathrm{mg}$, vitamin A $10.000 \mathrm{UI}$, vitamin D3 $2.500 \mathrm{UI}$, vitamin E $25 \mathrm{mg}$, vitamin K3 $2 \mathrm{mg}$. vitamin B1 $2.50 \mathrm{mg}$, vitamin B2 $6.50 \mathrm{mg}$, vitamin B6 $3.50 \mathrm{mg}$, vitamin B12 $18 \mathrm{mcg}$, folic acid $1.20 \mathrm{mg}$, pantothenic acid $15 \mathrm{mg}$, niacin $42 \mathrm{mg}$, biotin $80 \mathrm{mcg}$, ethoxyquin $166 \mathrm{mg}$

${ }_{2}^{2}$ Manganese $90 \mathrm{mg}$, zinc $75 \mathrm{mg}$, iron $60 \mathrm{mg}$, copper $9.75 \mathrm{mg}$, iodine $1.20 \mathrm{mg}$ 
To determine the biometry of the digestive tract, on the 21st day, one bird per replicate was identified and subjected to a six-hour fasting period, after which it was weighed and subsequently euthanized by cervical dislocation. Birds were eviscerated and organs were collected and weighed according to these steps: weight of the pro-ventricle, gizzard, pancreas weight, liver weight, small intestine weight, and large intestine weight. The values obtained were used to calculate the relative weight for each organ (RWO), through the equation:

$$
R W O(\%)=\left(\frac{\text { organ weight }}{\text { live weight }}\right) \times 100
$$

Data on metabolizability, performance, and biometry of the digestive tract were evaluated through an analysis of variance. When effects were deemed significant $(\alpha=0.05)$ Tukey's test was used to compare the means. All statistical analyses were performed using R software. The statistical model used was:

$$
y_{i j k}=m+a_{i}+b_{j}+(a b)_{i j}+e_{i j k}
$$

Where: $y_{i j k}=$ an observation from the $k^{\text {th }}$ replicate $(k=1,2, \ldots, 8)$,

$m=$ the overall mean,

$a_{i}=$ the fixed effect accounting for the addition of animal by-product meal,

$b_{i}=$ the fixed effect accounting for the addition of protease,

$(a b)_{i j}=$ the interaction effect of factors $a$ and $b$, and

$e_{i j k}=$ the random error with mean 0 and variance $\sigma^{2}$.

\section{Results and Discussion}

Significant differences were observed (Table 4) for ether extract metabolizability (EEMC) and balance (EEB), and for the metabolizability of nitrogen (NMC) and dry matter (DMMC). Treatments with animal-based meals showed the best values for the metabolizability coefficients. Nitrogen balance (NB) was did not differ across the treatments $(P>0.05)$. Interaction effects were observed $(P<0.05)$ between protein source and protease enzyme for EEMC and EEB. The plant-based protein source without protease supplementation decreased EEMC $(P<0.05)$ relative to the other treatments which were similar. Protease supplementation improved EEB when the protein source was plant based. However, protease supplementation decreased EEB when the protein source was animal based.

Means for performance attributes of broiler chicks fed diets supplemented with protease and animal by-product meal from 1 to seven days old are shown in Table 5. Birds that were fed animal-based meals grew more rapidly. Otherwise, no significant differences were found $(P>0.05)$.

Means for performance attributes of broiler chicks fed diets supplemented with protease and animal by-product meal from 1 to 21 days old are shown in Table 6 . Protease supplementation increased FI when the protein source was plant based. For birds fed animal-origin ingredients, the FI did not differ significantly whether or not protease was included in their diet, although the addition of protease did result in a numerical decrease in FI. For the remaining variables, no significant differences were found.

Means for performance attributes of broiler chicks fed diets supplemented with protease and animal by-product meal from 1 to 42 days old are shown in Table 7. Broilers fed diets with plant-based meals had better feed conversion (FC). Those birds that did not receive supplemental protease had improved liveability.

Means for relative weights of gastrointestinal organs of broiler chickens at 21 days old are shown in Table 8. Relative weight of the pancreas was higher in birds that received animal-based meals. Otherwise, no significant differences in relative weights of the gastrointestinal organs were observed. 
Table 4 Metabolizability (\%) of dry matter, nitrogen and ether extract by broiler chicks; and nitrogen and ether extract balances for feeds containing protease and animal by-product meal

\begin{tabular}{|c|c|c|c|c|c|}
\hline & NB (g) & EEB (g) & NMC (\%) & EEMC (\%) & DMMC (\%) \\
\hline \multicolumn{6}{|l|}{ Treatments } \\
\hline BVF & 29.35 & $43.34^{b}$ & 75.59 & $86.70^{\mathrm{b}}$ & 75.59 \\
\hline BFP & 27.26 & $48.66^{a}$ & 75.23 & $89.18^{a}$ & 75.23 \\
\hline BFA & 28.98 & $46.13^{a}$ & 79.34 & $90.88^{a}$ & 79.34 \\
\hline BFAP & 27.22 & $40.49^{b}$ & 79.29 & $90.10^{\mathrm{a}}$ & 79.29 \\
\hline \multicolumn{6}{|l|}{ Meals } \\
\hline Animal & 28.10 & $43.31^{b}$ & $79.32^{a}$ & $90.49^{a}$ & $79.32^{\mathrm{a}}$ \\
\hline Vegetable & 28.10 & $46.00^{\mathrm{a}}$ & $75.41^{b}$ & $87.94^{b}$ & $75.41^{b}$ \\
\hline \multicolumn{6}{|l|}{ Protease } \\
\hline Without & 29.17 & 44.73 & 77.47 & 88.79 & 77.57 \\
\hline With & 27.24 & 44.58 & 77.26 & 89.64 & 77.26 \\
\hline \multicolumn{6}{|l|}{$P$-value } \\
\hline Meals & 0.841 & 0.032 & 0.003 & $<0.001$ & 0.003 \\
\hline Protease & 0.059 & 0.896 & 0.866 & 0.166 & 0.866 \\
\hline Interaction & 0.866 & $<0.001$ & 0.903 & 0.011 & 0.903 \\
\hline$C V, \%$ & 9.83 & 7.57 & 4.39 & 1.90 & 2.99 \\
\hline
\end{tabular}

BVF: basal vegetable feed, BFP: BVF + protease, BFA: basal vegetable feed + animal by-product meal, BFAP: BFA + protease, DMMC: dry matter metabolizability, NMC: nitrogen metabolizability, EEMC: ether extract metabolizability, NB: nitrogen balance, EEB: ether extract balance

a,b Within a classification means with a common superscript do not differ at $P=0.05$

Table 5 Feed intake, average weight gain, final average weight, feed conversion and liveability of broiler chicks fed diets containing protease and animal by-product meal from 1 to 7 days old

\begin{tabular}{lccccc}
\hline & $\mathrm{Fl}, \mathrm{g}$ & AWG, & FAW, & FC, $\mathrm{kg} / \mathrm{kg}$ & Liveability, \% \\
\hline $\begin{array}{l}\text { Treatments } \\
\text { BVF }\end{array}$ & & & & & \\
BFP & 164.5 & 120.2 & 163.3 & 1.31 & 96.1 \\
BFA & 159.6 & 119.8 & 162.8 & 1.29 & 96.1 \\
BFAP & 159.2 & 122.2 & 164.8 & 1.24 & 96.1 \\
Meals & 160.8 & 124.2 & 167.2 & 1.26 & 96.6 \\
$\quad$ Animal & 160.0 & $123.2^{\mathrm{a}}$ & 166.0 & 1.252 & 96.3 \\
Vegetable & 162.1 & $120.0^{\mathrm{b}}$ & 163.0 & 1.302 & 96.1 \\
Protease & & & & & \\
With & 160.2 & 122.0 & 165.0 & 1.28 & 96.3 \\
$\quad$ Without & 161.9 & 121.2 & 164.1 & 1.28 & 96.1 \\
$P$-value & & & & & \\
Meals & 0.551 & 0.048 & 0.061 & 0.170 & 0.570 \\
Protease & 0.631 & 0.600 & 0.556 & 0.989 & 0.570 \\
Interaction & 0.349 & 0.430 & 0.335 & 0.647 & 0.570 \\
CV, \% & 4.54 & 2.81 & 2.04 & 5.74 & 1.23
\end{tabular}

BVF: basal vegetable feed, BFP: BVF + protease, BFA: basal vegetable feed + animal by-product meal, BFAP: BFA + protease, Fl: feed intake, AWG: average weight gain, FAW: final average weight, FC: feed conversion ratio

a,b Within a classification means with a common superscript do not differ at $P=0.05$ 
Table 6 Feed intake, average weight gain, final average weight, feed conversion and liveability of broilers fed diets containing protease and animal by-product meal from 1 to 21 days old

\begin{tabular}{|c|c|c|c|c|c|}
\hline & $\mathrm{FI}, \mathrm{g}$ & AWG, $g$ & FAW, $g$ & $\mathrm{FC}, \mathrm{kg} / \mathrm{kg}$ & Liveability, \% \\
\hline \multicolumn{6}{|l|}{ Treatments } \\
\hline BVF & $1259.7^{\mathrm{a}}$ & 776.4 & 822.3 & 1.46 & 92.9 \\
\hline BFP & $1295.8^{b}$ & 770.8 & 822.0 & 1.49 & 93.6 \\
\hline BFA & $1252.8^{\mathrm{ac}}$ & 776.2 & 839.5 & 1.45 & 92.8 \\
\hline BFAP & $1230.5^{c}$ & 765.0 & 813.0 & 1.44 & 94.2 \\
\hline \multicolumn{6}{|l|}{ Meals } \\
\hline Animal & $1241.7^{b}$ & 770.6 & 826.2 & 1.444 & 93.5 \\
\hline Vegetable & $1277.7^{\mathrm{a}}$ & 773.6 & 822.1 & 1.477 & 93.3 \\
\hline \multicolumn{6}{|l|}{ Protease } \\
\hline With & 1263.2 & 767.9 & 817.5 & 1.465 & 93.9 \\
\hline Without & 1256.3 & 776.3 & 830.9 & 1.457 & 92.9 \\
\hline \multicolumn{6}{|l|}{$P$-value } \\
\hline Meals & 0.019 & 0.702 & 0.458 & 0.149 & 0.537 \\
\hline Protease & 0.500 & 0.294 & 0.269 & 0.700 & 0.315 \\
\hline Interaction & 0.009 & 0721 & 0.294 & 0.445 & 0.396 \\
\hline$C V, \%$ & 1.96 & 2.35 & 1.37 & 3.33 & 1.16 \\
\hline
\end{tabular}

BVF: basal vegetable feed, BFP: BVF + protease, BFA: basal vegetable feed + animal by-product meal, BFAP: BFA + protease, Fl: feed intake, AWG: average weight gain, FAW: final average weight, FC: feed conversion ratio

a,b,c Within a classification means with a common superscript do not differ at $P=0.05$

Table 7 Feed intake, average weight gain, final average weight, feed conversion and liveability of broilers fed diets containing protease and animal by-product meal from 1 to 42 days old

\begin{tabular}{lccccc}
\hline & $\mathrm{Fl}, \mathrm{g}$ & AWG, $\mathrm{g}$ & $\mathrm{FAW}, \mathrm{g}$ & $\mathrm{FC}, \mathrm{kg} / \mathrm{kg}$ & Liveability, \% \\
\hline $\begin{array}{l}\text { Treatments } \\
\text { BVF }\end{array}$ & & & & & \\
BFP & 5184.2 & 2620.2 & 2748.3 & 1.71 & 92.9 \\
BFA & 5165.3 & 2610.8 & 2757.8 & 1.70 & 93.6 \\
$\quad$ BFAP & 5157.3 & 2608.7 & 2725.4 & 1.76 & 928 \\
Meals & 5045.8 & 2595.2 & 2737.0 & 1.75 & 94.2 \\
$\quad$ Animal & & & & & \\
$\quad$ Vegetable & 5101.6 & 2602.0 & 2731.2 & $1.76^{\mathrm{a}}$ & 93.3 \\
Protease & 5174.8 & 2615.5 & 2753.1 & $1.70^{\mathrm{b}}$ & 93.4 \\
$\quad$ With & & & & & \\
Without & 5105.6 & 2603.0 & 2747.4 & 1.730 & $93.8^{\mathrm{a}}$ \\
P-value & 5179.8 & 2614.5 & 2736.9 & 1.736 & $92.9^{\mathrm{b}}$ \\
$\quad$ Meals & & & & & \\
Protease & 0.208 & 0.646 & 0.520 & 0.032 & 0.909 \\
Interaction & 0.260 & 0.697 & 0.755 & 0.756 & 0.010 \\
CV, \% & 0.418 & 0.945 & 0.975 & 0.947 & 0.324 \\
& 2.29 & 2.26 & 2.48 & 3.33 & 1.16
\end{tabular}

BVF: basal vegetable feed, BFP: BVF + protease, BFA: basal vegetable feed + animal by-product meal, BFAP: BFA + protease, FI: feed intake, AWG: average weight gain, FAW: final average weight, FC: feed conversion ratio

a,b Within a classification means with a common superscript do not differ at $P=0.05$ 
Table 8 Relative weights of digestive system organs of broiler chickens at 21 days old when fed with diets containing protease and animal by-product meal

\begin{tabular}{lccccc}
\hline & Pancreas & Gizzard & Proventricle & Liver & Intestine \\
\hline $\begin{array}{l}\text { Treatments } \\
\text { BVF }\end{array}$ & & & & & \\
BFP & 0.28 & 2.50 & 0.52 & 2.64 & 7.48 \\
BFA & 0.34 & 2.44 & 0.52 & 2.67 & 7.37 \\
BFAP & 0.36 & 2.60 & 0.56 & 2.76 & 7.60 \\
Meals & 0.35 & 2.53 & 0.57 & 2.88 & 8.00 \\
Animal & & & & & \\
Vegetable & $0.36^{\mathrm{a}}$ & 2.57 & 0.56 & 2.82 & 7.80 \\
Protease & $0.31^{\mathrm{b}}$ & 2.47 & 0.52 & 2.66 & 7.43 \\
With & & & & & \\
Without & 0.35 & 2.49 & 0.55 & 2.78 & 7.68 \\
$P$-value & 0.32 & 2.55 & 0.54 & 2.70 & 7.54 \\
Meals & & & & & \\
Protease & 0.018 & 0.520 & 0.205 & 0.132 & 0.312 \\
Interaction & 0.173 & 0.658 & 0.856 & 0.490 & 0.691 \\
\hline BVF: basalvegetab & 0.053 & 0.996 & 0.847 & 0.645 & 0.477 \\
\hline
\end{tabular}

BVF: basal vegetable feed, BFP: BVF + protease, BFA: basal vegetable feed + animal by-product meal, BFAP: BFA + protease

a,b Within a classification means with a common superscript do not differ at $P=0.05$

The present results for metabolizability may be compared with those of Laboissière (2008), who evaluated viscera and bone meals with different levels of humidity in the processing of broiler feed, and found that treatments with those meals showed improved results for DMMC and NMC. Contrary to the results that are presented here, Brito et al. (2006) observed 6\% improvement in EEMC when they added a multi-enzymatic complex containing protease to broiler diets based on extruded soybean.

Laboissière (2008) also found better performance in seven-day-old pre-initial broilers fed viscera and bone meals. The results of the present study were similar to those observed by Bellaver et al. (2005), who compared the inclusion of $3 \%$ of viscera and bone meal in broiler diets with vegetable diets and observed no effects on performance at 21 days old.

These results differ from those found by Guichard and Djakalia (2008), who evaluated the substitution of animal-origin meals with plant-origin ones in diets for broiler chickens and found that animal by-product meals resulted in better feed conversions and higher weight gains in the same period. A possible explanation can be related to the origins and processing methods of the animal meal. Troni et al. (2016) stated that during the production of animal meal for the poultry industry, the inclusion of sources of by-products such as feathers, blood, and viscera may occur and can contribute to the nutrient level variability between these alternative feedstuffs.

In terms of protease use, better performance was expected for animals that received a supplemented diet, since protease supplementation enhances the digestibility of proteins and amino acids. Therefore, endogenous enzyme production is optimized, thus helping to improve ingredient quality, reducing their variability and ameliorating negative effects of trypsin inhibitors (Cowieson et al., 2016; Walk et al., 2018). Park and Kim (2018) observed that broilers fed diets supplemented with protease had better weight gain and feed conversion compared with birds fed diets without it. Furthermore, Freitas et al. (2011) evaluated a serine monocomponent protease and found better weight gain and feed conversion for birds fed diets containing protease compared with those with no enzymatic supplementation. On the other hand, Park and Kim (2018) did not observe any influence on seven-day-old broiler performance with feed containing protease combined with essential oils. Nor did Zotesso (2015) did not observe any effect of including protease on performance variables for broiler chickens between 1 and 42 days old.

Protease was expected to influence pancreas biometry because of the increased production of endogenous enzymes. Fidelis et al. (2011) reported that protease increased the effects of endogenous 
pancreatic enzymes, augmenting the hydrolysis and solubilization of the protein. However, in the present study, even though the authors observed the influence of diets on pancreas biometry, this related to the type of meal used and not to protease use. Although no protease influence on the organ biometry was observed, the results are consistent with those of Park and Kim (2018), who did not observe significant differences in relative organ weight in chickens fed diets with protease supplementation.

\section{Conclusions}

Use of animal by-product meal as a source of protein in the starter feed of broiler chickens improved digestibility and performance of broiler chicks. Use of protease during the starter rearing period is recommended mainly for vegetable-based diets.

\section{Acknowledgements}

This study was financed in part by the Coordenação de Aperfeiçoamento de Pessoal de Nível Superior - Brazil (CAPES) - Finance Code 001.

\section{Authors' Contributions}

DPC, NSML and JHS conceived and designed the experiments. DPC, HFO, MFP, KAT and PSA performed the experiments. MFP analysed the data. MAA, NSML and JHS contributed reagents, materials, and analytical tools. DPC and HFO wrote the paper. HFO edited the manuscript.

\section{Conflicts of Interest Declaration}

The authors declare they have no conflicts of interest regarding the work presented in this report.

\section{References}

Batal, A.B. \& Parsons, C.M., 2002. Effects of age on nutrient digestibility in chicks fed different diets. Poult. Sci. 81, 400407. https://doi.org/10.1093/ps/81.3.400

Bellaver, C., Costa, C.A.F., Avila, V.S., Fraha, M., Lima, G.J.M.M., Hackenhar, L. \& Baldi, P., 2005. Substituição de farinhas de origem animal por ingredientes de origem vegetal em dietas para frangos de corte. Cienc. Rural 35 , 671-677. http://dx.doi.org/10.1590/S0103-84782005000300030

Brazilian Association of Animal Protein (ABPA). Annual Activity Report, 2018. São Paulo, Brazil. Accessed 15 April 2019. http://abpa-br.org/wp-content/uploads/2018/10/relatorio-anual-2018.pdf

Brito, C.O., Albino, L.F.T., Rostagno, H.S., Gomes, P.C., Dionízio, M.A. \& Carvalho, D.C.O., 2006. Adição de complexo multienzimático em dietas à base de soja extrusada e desempenho de pintos de corte. Rev. Bras. Zootec. 35, 457-641. http://dx.doi.org/10.1590/S1516-35982006000200017

Cowieson, A.J., Lu, H., Ajuwon, K.M., Knap, I. \& Adeola, O., 2016. Interactive effects of dietary protein source and exogenous protease on growth performance, immune competence and jejunal health of broiler chickens. Anim. Prod. Sci. 57, 252-261. https://doi.org/10.1071/AN15523

Fidelis, F.N., Kluenter, A.M., Fischer, M. \& Pontoppidan, K., 2011. A feed serine protease improves broiler performance and increases protein and energy digestibility. J. Poult. Sci. 48, 239-246. https://doi.org/10.2141/jpsa.011035

Freitas, D.M., Vieira, S.L., Angel, C.R., Favero, A. \& Maiorka, A., 2011. Performance and nutrient utilization of broilers fed diets supplemented with a novel mono-component protease. J. Appl. Poult. Res. 20, 322-334. https://doi.org/10.3382/japr.2010-00295

Ghazi, S., Rooke, J.A., Galbraith, H. \& Bedford, M.R., 2002. The potential for the improvement of the nutritive value of soya-bean meal by different proteases in broiler chicks and broiler cockerels. Br. Poult. Sci. 43, 70-77. https://doi.org/10.1080/00071660120109935

Guichard, B.L. \& Djakalia, B., 2008. Effect of feather meal feeding on the body weight and feather development of broilers. Eur. J. Sci. Res. 24, 404-409.

Laboissière, M., 2008. Farinhas de resíduos de abatedouros avícolas em diferentes graus de processamento em rações pré-iniciais e iniciais de frangos de corte. [Master of Animal Science]; 1-70. Universidade Federal de Goiás, Goiânia, Brazil.

Matias, C.F.Q., Rocha, J.S.R., Pompeu, M.A., Baião, R.C., Baião, N.C., Lara, L.J.C., Clímaco, W.L.S., Pereira, L.F.P., Caldas, E.O., Teixeira, M.P.F. \& Cardeal, P.C., 2015. Efeito da protease sobre o coeficiente de metabolizabilidade dos nutrientes em frangos de corte. Arq. Bras. Med. Vet. Zootec. 67, 492-498. http://dx.doi.org/10.1590/1678-7883

Matterson, L.D., Potter, L.M., Stutz, M.W. \& Singsen, E.P., 1965. The metabolizable energy of feed ingredients for chickens. Res. Rep. Conn. Agric. Exp. Stn. 7, 1-11.

Oliveira, H.F., Souto, C.N., Di Castro, I.C., Mello, H.H.C. \& Mascarenhas, A.G., 2018. Agroindustrial wastes of fruit processing in broilers feeding: review. Rev. Port. Ciên. Vet. 113, 1-10.

Park, J.H. \& Kim, I.H., 2018. Effects of a protease and essential oils on growth performance, blood cell profiles, nutrient retention, ileal microbiota, excreta gas emission, and breast meat quality in broiler chicks. Poult. Sci. 0, 1-7. https://doi.org/10.3382/ps/pey151

Ribeiro, J.S., Fassani, E.J., Makiyama, L. \& Clemente, A.H.S., 2015. Suplementação de enzimas amilase, fitase e protease para codornas japonesas em postura. Bol. Ind. Anim. 72, 163-169. http://dx.doi.org/10.17523/bia.v72n2p163 
Rostagno, H.S., Albino, L.F.T., Donzele, J.L., Gomes, P.C., Oliveira, R.F., Lopes, D.C., Ferreira, A.S., Barreto, S.L.T. \& Euclides, R.F., 2011. Tabelas brasileiras para aves e suínos: composição de alimentos e exigências nutricionais. Editora da Universidade Federal de Viçosa, Minas Gerais, Brazil.

Sakomura, N.K. \& Rostagno, H.S., 2007. Métodos de pesquisa em nutrição de monogástricos. Funep, São Paulo, Brazil.

Silva, D.J. \& Queiroz, A.C., 2002. Análise de alimentos (métodos químicos e biológicos). Editora da Universidade Federal de Viçosa, Minas Gerais, Brazil.

Troni, A.R., Gomes, P.C., Mello, H.H.C., Albino, L.F.T. \& Rocha, T.C., 2016. Composição química e energética de alimentos para frangos de corte. Rev. Ciên. Agron. 47, 755-760. http://dx.doi.org/10.5935/1806-6690.20160090

Wachholz, L., Vianna, R.N., Broch, J. \& Souza, C., 2017. Possibilidade do uso de mexilhão dourado contaminado com metais tóxicos em dietas para frangos de corte. Rev. Colomb. Cienc. Anim. 9, $227-235$. http://dx.doi.org/10.24188/recia.v9.n2.2017.562

Walk, C.L., Pirgozliev, V., Juntunen, K., Paloheimo, M. \& Ledoux, D.R., 2018. Evaluation of novel protease enzymes on growth performance and apparent ileal digestibility of amino acids in poultry: Enzyme screening. Poult. Sci. 0, 116. https://doi.org/10.3382/ps/pey080

Zotesso, F., 2015. Efeito de diferentes níveis proteicos e inclusões de protease sobre o desempenho de frango de corte. [Master of Animal Science] 1-93. Universidade de São Paulo, Pirassununga, Brazil. 